\title{
Sports Management Education in Turkish Universities, Analysis of Undergraduate Programs
}

\author{
Elif Bozyigit \\ Department of Sport Management, Faculty of Sport Sciences, Pamukkale University, Denizli 20070, Turkey
}

\begin{abstract}
The aim of the study is to examine the undergraduate program courses of the universities providing training in Sports Management in Turkey. In this study, electronic screening method was used via web sites of universities. 80 Sports Management Departments (SMD) were found and only 20 of them which were up to date were analyzed. 2016-2017 academic year curriculums were examined. All universities examined were state universities. The courses were grouped as Compulsory-Elective, Theoretical-Practical and Total number of course-hour and credit. The percentages of the courses were calculated over the total course hours for that group.
\end{abstract}

Key words: Sports management, education, curricula, university, Turkey.

\section{Introduction}

Sports management education programs are available all over the World. However, these educations vary according to the educational strategies of the countries and the needs in the area. Training in Sports Manager in Turkey is only provided by state and private universities. The education of state universities is free.

According to the definition of Turkish Employment Agency, the sports manager is the person responsible for the sports services and activities [1]. Sports Management Education Programs in universities prepare students for careers in the sports management. For example; sports marketing, Sports economics, sports business, media and sport, sports organizations and sports facilities, contemporary developments, etc.

\section{Materials and Methods}

In this study, electronic screening method was used via official websites of universities. 80 Sports Management Departments (SMD) were found [2] and only 20 of them which were up to date were analyzed.

Corresponding author: Elif Bozyigit, Ph.D., assistant professor, research fields: sports management, mobbing, leadership.
2016-2017 academic year curriculums were examined. All universities examined were state universities. The courses were grouped as theoretical, practical and total compulsory-elective course hours. Total credit for all course groups was calculated and European Credit Transfer System (ECTS) value was also added to table. Percentage values of the courses were calculated over the total course hours for that group.

\section{Results}

The total number of courses studied in the research is 1156. The number of courses in the curriculums was at least 40 (ANDU) and at most 74 (AKDU). Theoretical course hours were minimum 107 (OMU), maximum 165 (AKDU). Practical course hours were minimum 8 (FU), maximum 72 (ANKU) (Table 1).

In general, it has been determined that the compulsory courses consist of field knowledge (sports management, general business, economics, law etc.) and sports branches (athletics, gymnastics, swimming, volleyball, etc.) Sample semester plans can be seen in Table 2.

\section{Discussion}

The sport manager in Turkey is trained for 4 years (8 semesters) with a focus on vocational courses. This 
Table 1 Course distributions of curriculums.

\begin{tabular}{|c|c|c|c|c|c|c|c|c|c|c|c|c|c|c|c|c|c|c|c|c|c|c|c|}
\hline & \multirow{2}{*}{ Universities } & \multicolumn{7}{|c|}{ Total Compulsory Course Hours } & \multicolumn{7}{|c|}{ Total Elective Course Hours } & \multicolumn{8}{|c|}{ CourseTotal Hours } \\
\hline & & $n$ & Total & $\mathrm{T}$ & $\%$ & $\mathrm{P}$ & $\%$ & $\mathrm{C}$ & $n$ & Total & $\mathrm{T}$ & $\%$ & $\mathrm{P}$ & $\%$ & $\mathrm{C}$ & $n$ & Total & $\mathrm{T}$ & $\%$ & $\mathrm{P}$ & $\%$ & $\mathrm{TC}$ & ECTS \\
\hline 1 & IBU [3] & 40 & 125 & 113 & 90 & 12 & 10 & 119 & 6 & 18 & 18 & 100 & 0 & 0 & 18 & 46 & 143 & 131 & 92 & 12 & 8 & 137 & 240 \\
\hline 2 & ADU [4] & 55 & 157 & 125 & 80 & 32 & 20 & 141 & 9 & 18 & 18 & 100 & 0 & 0 & 18 & 64 & 175 & 143 & 82 & 32 & 18 & 159 & 240 \\
\hline 3 & AKDU [5] & 54 & 142 & 126 & 89 & 16 & 11 & 132 & 20 & 55 & 39 & 71 & 16 & 29 & 47 & 74 & 197 & 165 & 84 & 32 & 16 & 179 & 240 \\
\hline 4 & ANDU [6] & 52 & 140 & 132 & 94 & 8 & 6 & 202 & 13 & 11 & 5 & 45 & 6 & 55 & 38 & 40 & 151 & 137 & 91 & 14 & 9 & 240 & 240 \\
\hline 5 & ANKU [7] & 56 & 165 & 103 & 62 & 62 & 38 & 134 & 15 & 23 & 13 & 57 & 10 & 43 & 21 & 71 & 159 & 116 & 73 & 72 & 45 & 155 & 241 \\
\hline 6 & ATAU [8] & 53 & 181 & 137 & 76 & 44 & 24 & 147 & 3 & 6 & 6 & 100 & 0 & 0 & 6 & 56 & 187 & 143 & 76 & 44 & 24 & 153 & 240 \\
\hline 7 & BAU [9] & 47 & 127 & 118 & 93 & 9 & 7 & 173 & 14 & 41 & 39 & 95 & 2 & 5 & 66 & 61 & 168 & 157 & 93 & 11 & 7 & 239 & 239 \\
\hline 8 & DU [10] & 52 & 136 & 119 & 88 & 17 & 13 & 140 & 14 & 50 & 38 & 76 & 12 & 24 & 50 & 66 & 186 & 157 & 84 & 29 & 16 & 190 & 240 \\
\hline 9 & FU [11] & 45 & 127 & 119 & 94 & 8 & 6 & 123 & 8 & 24 & 24 & 100 & 0 & 0 & 24 & 53 & 151 & 143 & 95 & 8 & 5 & 147 & 240 \\
\hline 10 & GAZIU [12] & 43 & 134 & 118 & 88 & 16 & 12 & 186 & 10 & 38 & 30 & 79 & 8 & 21 & 54 & 53 & 172 & 148 & 86 & 24 & 14 & 240 & 240 \\
\hline 11 & IU [13] & 52 & 163 & 129 & 79 & 34 & 21 & 143 & 7 & 14 & 14 & 100 & 0 & 0 & 14 & 59 & 177 & 143 & 81 & 34 & 19 & 157 & 240 \\
\hline 12 & KATU [14] & 46 & 138 & 122 & 88 & 16 & 12 & 189 & 12 & 27 & 17 & 63 & 10 & 37 & 45 & 58 & 165 & 139 & 84 & 26 & 16 & 234 & 234 \\
\hline 13 & MCBU [15] & 39 & 115 & 97 & 84 & 18 & 16 & 185 & 16 & 42 & 28 & 67 & 14 & 33 & 53 & 55 & 157 & 125 & 80 & 32 & 20 & 238 & 240 \\
\hline 14 & MU [16] & 51 & 127 & 101 & 80 & 26 & 20 & 201 & 13 & 30 & 22 & 73 & 8 & 27 & 39 & 64 & 157 & 123 & 78 & 34 & 22 & 240 & 240 \\
\hline 15 & MSKU [17] & 47 & 149 & 129 & 87 & 20 & 13 & 178 & 12 & 38 & 31 & 82 & 7 & 18 & 62 & 59 & 187 & 160 & 86 & 27 & 14 & 240 & 240 \\
\hline 16 & OMU [18] & 50 & 111 & 101 & 91 & 10 & 9 & 151 & 8 & 8 & 6 & 75 & 2 & 25 & 25 & 58 & 119 & 107 & 90 & 12 & 10 & 176 & 188 \\
\hline 17 & OHU [19] & 41 & 122 & 107 & 88 & 15 & 12 & 175 & 13 & 40 & 31 & 78 & 9 & 23 & 65 & 54 & 162 & 138 & 85 & 24 & 15 & 240 & 240 \\
\hline 18 & PAU [20] & 50 & 136 & 98 & 72 & 38 & 28 & 167 & 14 & 36 & 36 & 100 & 0 & 0 & 46 & 64 & 172 & 134 & 78 & 38 & 22 & 240 & 240 \\
\hline 19 & SAU [21] & 31 & 115 & 102 & 89 & 13 & 11 & 117 & 15 & 56 & 52 & 93 & 4 & 7 & 56 & 46 & 171 & 154 & 90 & 17 & 10 & 173 & 240 \\
\hline 20 & SEU [22] & 43 & 124 & 102 & 82 & 22 & 18 & 189 & 12 & 29 & 24 & 83 & 5 & 17 & 36 & 55 & 153 & 126 & 82 & 27 & 18 & 225 & 225 \\
\hline
\end{tabular}

T: Theoretical, P: Practical, C: Credit, TC: Total Credit, ECTS: European Credit Transfer System. 
Table 2 Sample Semesters Plans (3rd Semester).

\begin{tabular}{|c|c|c|c|c|c|c|c|c|c|}
\hline Course Title (PAU) & $\mathrm{T}$ & $\mathrm{P}$ & ECTS & Type & Course Title (BAU) & $\mathrm{T}$ & $\mathrm{P}$ & ECTS & Type \\
\hline Basic Computer Sciences & 2 & 2 & 4 & Comp. & Gymnastics & 3 & 2 & 6 & Comp. \\
\hline $\begin{array}{l}\text { Contact Between Individuals } \\
\text { and Organizations }\end{array}$ & 2 & 0 & 4 & Comp. & Introduction to Sociology & 2 & 0 & 3 & Comp. \\
\hline Sports Management & 2 & 0 & 4,5 & Comp. & Introduction to Law & 2 & 0 & 3 & Comp. \\
\hline $\begin{array}{l}\text { Read and Speak in Foreign } \\
\text { Lang. }\end{array}$ & 2 & 0 & 3 & Comp. & Sport Management - I & 3 & 0 & 4 & Comp. \\
\hline $\begin{array}{l}\text { Ataturk Principles and History } \\
\text { of Turkish Revolution-I }\end{array}$ & 2 & 0 & 2 & Comp. & Sports Physiology & 3 & 0 & 6 & Comp. \\
\hline Volleyball & 1 & 2 & 3 & ODC & Individual Sports III (Table Tennis) & 3 & 0 & 4 & Elec. \\
\hline II. Foreign Language-I & 2 & 0 & 3 & Elec. & Individual Sports III (Bocce) & 3 & 0 & 4 & Elec. \\
\hline OFEF -eg: Sport Tourism & 2 & 0 & 3 & Elec. & Team Sports III (Basketball) & 3 & 0 & 4 & Elec. \\
\hline FE-eg: Media and Sports & 3 & 0 & 3,5 & Elec. & & & & & \\
\hline
\end{tabular}

Comp: Compulsory, Elec: Elective, ODC: Out of Department Comp., OFEF: Out of Field Elective Fall, FE: Fall Ellective.

training consists of theoretical and practical courses.

Besides, these courses are divided into two as compulsory and elective courses. In addition, students must complete a two-semester internship (7th and 8th semester). Theoretical courses consist of courses such as effective written and verbal communication skills and at least two languages (elective-English, German and French)

\section{Conclusions}

The course period of SMD is 8 semesters including fall and spring semesters, 4 years in total.

Curriculums are independent of each other and are compatible with ECTS.

Courses are taught as theoretical-practical and compulsory-elective.

In the majority of the curriculums, it has been found that the practical courses are given only in theory.

Most of the Sports Science faculties carry out a Special Ability Test at the entrance to the school. For this reason, curriculums are thought to be both theoretical and practical.

\section{Acknowledgments}

This study was presented as a poster presentation at the 2nd World Association for Sport Management World Conference (June 20-23, 2017, Kaunas, Lithuania).

\section{References}

[1] Turkish Employment Agency. 2017. Professional Knowledge Bank. Accessed November 04, 2017. http://e-ogrenme.iskur.gov.tr/oyscontent/Courses/Course1 62/pdf/s/69.pdf (in Turkish).

[2] Council of Higher Education. 2017. Accessed February 11, 2017. https://istatistik.yok.gov.tr/

[3] Abant Izzet Baysal University (IBU) Curriculum, 2017-2018. Accessed February 12, 2017. http://besyo.ibu.edu.tr/tr/spor-yoneticiligi-mufredati

[4] Adnan Menderes University (ADU) Curriculum, 2017-2018. Accessed February 12, 2017. http://www.akademik.adu.edu.tr/yo/besyo/default.asp?idx $=313337$

[5] Akdeniz University (AKDU) Curriculum, 2017-2018. Accessed $\quad$ February 2017. http://dersbilgipaketi.akdeniz.edu.tr/tr-TR/Program/Brow se/1003/1058

[6] Anadolu University (ANDU) Curriculum, 2017-2018. Accessed $\quad$ February 2017. http://abp.anadolu.edu.tr/tr/program/dersler/1860/13

[7] Ankara University (ANKU) Curriculum, 2017-2018. Accessed $\quad$ February 2017. http://sporyoneticiligi.sporbilimleri.ankara.edu.tr/tr/ders-p rogrami/

[8] Ataturk University (ATAU) Curriculum, 2017-2018. $\begin{array}{lll}\text { Accessed } & \text { February } & 12,\end{array}$ https://atauni.edu.tr/spor-yoneticiligi-bolumu-1

[9] Balikesir University (BAU) Curriculum, 2017-2018. $\begin{array}{lll}\text { Accessed } & \text { February } & 12,\end{array}$ https://obs.balikesir.edu.tr/oibs/bologna/start.aspx?gkm=0 2423330037707333423223035540377073557034184376 79366903889631120 
[10] Duzce University (DU) Curriculum, 2017-2018. Accessed February 12 , 2017. http://sbf.duzce.edu.tr/Sayfa/4566/ders-programlari

[11] Firat University (FU) Curriculum, 2017-2018. Accessed February 12, 2017. http://sb.firat.edu.tr/tr/node/137

[12] Gazi University (GAZIU) Curriculum, 2017-2018. Accessed $\quad$ February 2017. http://sporbilimleri.gazi.edu.tr/posts/view/title/spor-yoneti ciligi-2814

[13] Inonu University (IU) Curriculum, 2017-2018. Accessed February 12 , 2017 https://cms.inonu.edu.tr/tr/cms/syb/dersler/232

[14] Karadeniz Teknik University (KATU) Curriculum, 2017-2018. Accessed February 12, 2017. http://www.ktu.edu.tr/syb

[15] Manisa Celal Bayar University (MCBU) Curriculum, 2017-2018. Accessed February 12, 2017. http://katalog.cbu.edu.tr/Site/CourceStructure.aspx?Progr amID=292\&lang=1

[16] Marmara University (MU) Curriculum, 2017-2018. Accessed $\quad$ February 2017. http://llp.marmara.edu.tr/organizasyon.aspx?kultur=tr-TR $\&$ Mod=1\&ustbirim=3050\&birim=3053\&altbirim=\&prog ram $=117 \&$ organizasyonId $=628 \&$ mufredatTurId=932004
\#Anchor3

[17] Mugla Sitki Kocman University (MSKU) Curriculum, 2017-2018. Accessed February 12, 2017. http://sporyoneticiligi.mu.edu.tr/tr/mufredat-2886

[18] Ondokuz Mayis University (OMU) Curriculum, 2017-2018. Accessed February 12, 2017. http://ebs.omu.edu.tr/ebs/program.php?dil=tr\&mod=1\&P rogram $=2767$

[19] Omer Halisdemir University (OHU) Curriculum, 2017-2018. Accessed February 12, 2017 https://www.ohu.edu.tr/besyo/sporyoneticiligi/dersplani

[20] Pamukkale University (PAU) Curriculum, 2017-2018. Accessed $\quad$ February 2017. http://ebs.pau.edu.tr/BilgiGoster/Program.aspx?lng=1\&dz $\mathrm{y}=3 \& \mathrm{br}=416 \& \mathrm{bl}=510 \& \mathrm{pr}=360 \& \mathrm{dm}=1 \& \mathrm{ps}=2 \#$ dersPlanA KTS

[21] Sakarya University (SAU) Curriculum, 2017-2018. Accessed $\quad$ February 2017. http://ebs.sabis.sakarya.edu.tr/DersPlan/tr/2016/20905/22 12/0/0/0\#3

[22] Selcuk University (SEU) Curriculum, 2017-2018. $\begin{array}{lll}\text { Accessed } & \text { February } & 12,\end{array}$ https://www.selcuk.edu.tr/besyo/spor_yoneticiligi/bolum _dersleri/tr 